\title{
Impact of cataract surgery on car driving: a population based study in Sweden
}

\author{
Eva Mönestam, Lillemor Wachtmeister
}

\begin{abstract}
Aims-To investigate the outcome of cataract surgery on the patients' self estimation of visual function while driving. Furthermore, the benefit of surgery to the car driving population was determined. Methods-A total of 208 consecutive patients (211 cases) with driving licences, who underwent cataract surgery with intraocular lenses, were studied prospectively using self administered questionnaires. Their self estimated degree of visual functional problems while driving were analysed before and after surgery.

Results-Visual problems while driving declined from $82 \%$ preoperatively to $5 \%$ after surgery. Remaining visual problems with the operated eye were present in only seven patients. Problems in estimating distance while driving decreased from $37 \%$ before surgery to $6 \%$ after surgery. Twenty three per cent of the patients drove with a visual acuity below the requirements for driving in Sweden before surgery and only $4 \%$ after surgery. There was no correlation between the degree of visual problems while driving and visual acuity before surgery.

Conclusion-The car driving population greatly benefited from cataract surgery in terms of subjectively improved visual function and distance estimation while driving. Functional visual problems while driving should be considered as an indication for cataract surgery. These findings also indicated that the second eye should be operated on, if necessary, to achieve optimal ability to estimate distance and give best possible road safety.

(Br F Ophthalmol 1997;81:16-22)
\end{abstract}

Driving is a highly visual task. Approximately $90 \%$ of the information used while driving is visual. ${ }^{1}$ Visual deficit in central and peripheral vision has been shown to be significantly correlated with increased frequency of crashes. ${ }^{2-4}$ Recently, it has also been reported that visual attention and mental status are variables that can significantly predict frequency of crashes. ${ }^{3}$ Visual attention and visual acuity are highly related to the health of the eye and the visual sensory function. ${ }^{3}$ As the mean reaction time is longer in old age, ${ }^{5}$ optimal visual performance is especially important for the elderly. ${ }^{6}$

Several studies concerning visual acuity results as the primary outcome of cataract surgery have been presented. ${ }^{7}$ However, it is well known that other factors such as glare sensitivity, contrast sensitivity, night vision, and visual fields are salient for the total visual function. ${ }^{9}$ Only a few studies have focused on the patients' own assessment of visual function, before and after cataract surgery. ${ }^{10}$ To our knowledge, the impact of cataract surgery on the ability to drive, from the patients' point of view, is unknown. The present study examined the outcome of cataract surgery on patients' self estimation of visual function while car driving as well as their visual acuity. Furthermore, we also attempted to determine the benefit of cataract surgery for the car driving population.

\section{Patients and methods}

Between 1 April 1992 and 31 March 1993 all patients who underwent cataract surgery at Norrlands University Hospital in Umeå, Sweden were registered. All cataract surgery in this region is performed at the eye clinic of the university hospital. The admitting area has a population of about 175000 people. ${ }^{11}$ This region is sparsely populated, with only 3.8 inhabitants per square kilometre. The frequency of cataract surgery was 3.3 per 1000 population for the 1 year period studied.

A total of 572 patients were registered; patients who underwent cataract surgery for reasons other than restoring vision or had traumatic, juvenile, or congenital cataracts were excluded $(n=33)$. Also excluded were 14 patients whose mental status was too poor to allow them to cooperate with a questionnaire and 11 patients who were preoperatively scheduled for cataract surgery without an IOL implantation. Patients with combined cataract and corneal or trabeculectomy surgery were not included in the study $(n=16)$.

The study comprised of 498 patients, a total of 504 senile and presenile cataract cases. Three patients were withdrawn from the study because of severe complications occurring before the second questionnaire had been answered; one developed rapidly progressing neovascular glaucoma shortly after the cataract surgery. In spite of intensive laser treatment it was necessary to implant a Molteno tube within 3 months after the cataract surgery. The second patient had a low grade endophthalmitis postoperatively which made it necessary to explant the IOL 2 months after the cataract surgery. The third patient had a perforating injury by the anaesthetic needle. Eighteen patients died during the follow up period and
Accepted for publication 28 June 1996 

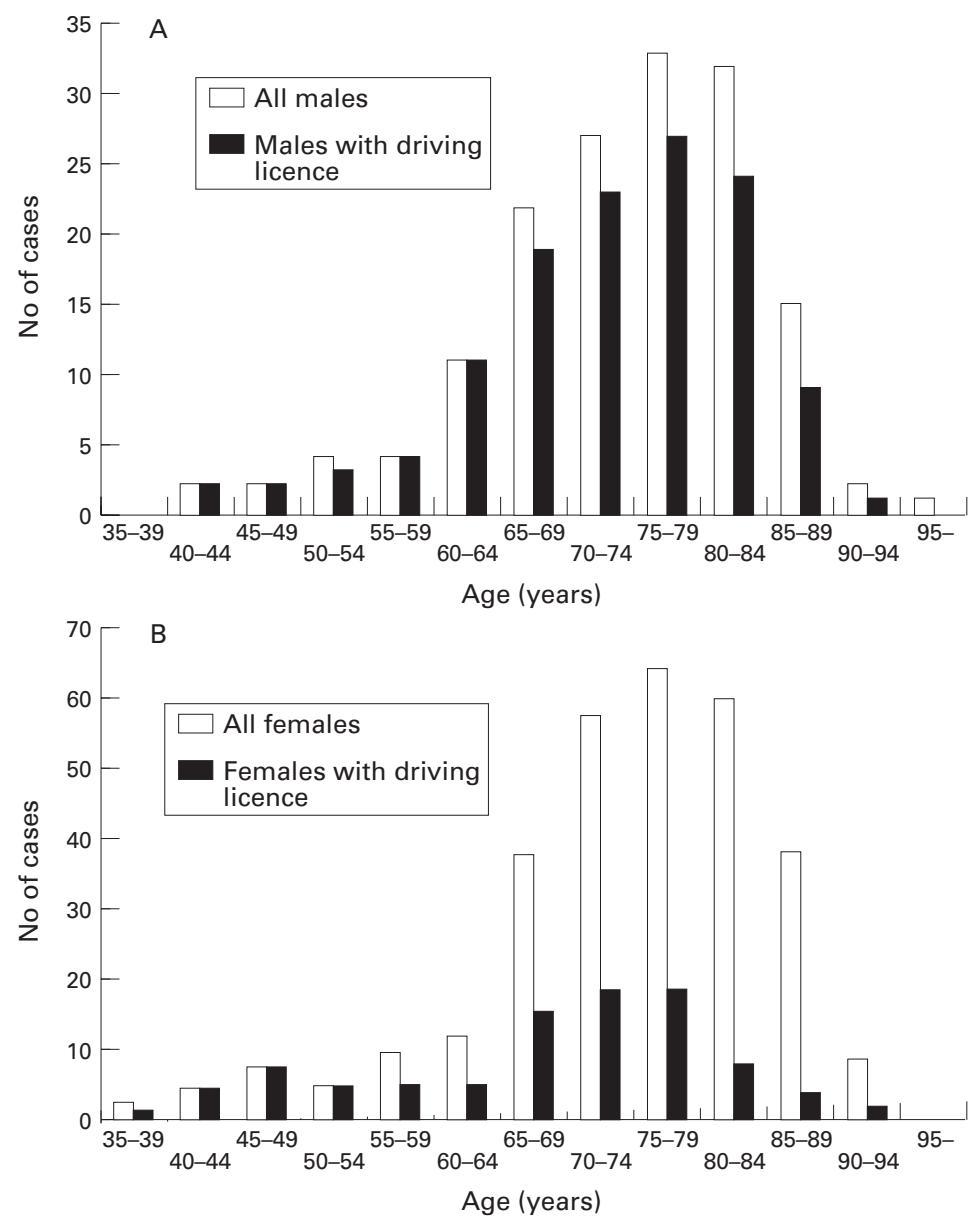

Figure 1 Distribution of driving licences in men $(A)$ and women (B) of different ages.

24 were excluded because of missing data. The dropout frequency was $8.9 \%$ (45/504). No significant differences in age and sex were found between the dropouts and those included. A total of 459 surgical events in 453 patients were finally included in the study; six patients $(1 \%)$ had surgery on both eyes.

Self administered questionnaires were used; they were intentionally designed to include only a reasonable number of simple questions as they were to be addressed to elderly people. They aimed to obtain, with a good response rate, self estimated changes in visual function before compared with after cataract surgery. The questions concerned reading ability, television viewing, mobility, and car driving. Identical or very similar questions have been used previously in well validated questionnaires of high reliability concerning visual function and impairment in subjects with cataracts. ${ }^{12} 13$ The questions analysed in the present study are presented in the appendix.

A few days before surgery the questionnaire was mailed to the patient. On the day of cataract surgery the questionnaire was delivered by the patient to the nursing staff of the eye clinic. It was checked that the questions had been understood and completely answered.

The type of surgery performed was a standard extracapsular cataract extraction (ECCE) in 390 cases $(85 \%, 299$ first eyes and 91 second eyes), phacoemulsification (PE) in 57 cases $(12 \%, 47$ first eyes and 10 second eyes), and intracapsular cataract extraction (ICCE) in 12 cases ( $3 \%$, seven first eyes and five second eyes). The complication rates concerning zonular/posterior capsular rupture with or without vitreous loss were $5.6 \%$ for ECCE and $3.5 \%$ for PE; most of them were minor. Only $2.6 \%(12 / 447)$ of all ECCE and PE cases received an anterior chamber IOL.

Two to 3 months after surgery the patients' records were studied to obtain information about the best corrected monocular visual acuity and refraction of both eyes before and after surgery. It was ensured that the eye had healed, vision had stabilised, and that glasses had been prescribed if necessary.

One to 2 months after the patients had received their prescription glasses, a second questionnaire was mailed to them (see appendix). The mean time lapse from date of surgery to when the second questionnaire was returned was $5.3(\mathrm{SD}=1.8)$ months. This delay was to give the patients the time necessary to adjust to their new glasses.

Patients with driving licences were identified in the Swedish National Register of Driving Licences; 208 patients (211 cases) had licences and 245 patients (248 cases) had not. None of the patients without driving licences drove before or after surgery. If it was not clear from the questionnaire why the patients had stopped driving, additional data were collected by telephone.

REQUIREMENTS FOR DRIVING

In Sweden the requirements for noncommercial car driving licences are: visual acuity binocularly with best correction of at least logMAR (minimum angle of resolution) 0.3 (20/40) and binocular visual fields equal to at least the normal visual field size of one eye.

\section{STATISTICAL METHODS}

Distance acuity for letters was expressed in terms of $\log M A R$. For statistical purposes a visual acuity of $\operatorname{logMAR}>1.7(<2 / 100)$ was considered $\log M A R=2(=2 / 200)$, when tabulating the data. Paired two sample, two tailed $t$ tests were used to analyse the improvement in visual acuity after surgery. One way analysis of variance was used to compare age differences in the various subgroups. Yates's corrected $\chi^{2}$ test or Fisher's exact test was used to analyse the two by two frequency tables.

\section{Results}

DRIVING LICENCES AND SEX AND AGE

DISTRIBUTIONS

Of the 459 surgical events included in the study, 155 were in men (34\%) and 304 in women $(66 \%)$. The distributions of age for men and women are shown in Figures $1 \mathrm{~A}$ and B. The median ages for men and women were 75 and 77 years, respectively. The age range was 38-95 years.

A total of 211 surgical cases ( $46 \%$ ) had driving licences; 125 (59\%) were men and 86 
Table 1 Self estimated visual functional problems while driving before surgery in relation to age, sex, and surgery on the first and the right eye, respectively, in cases with driving licences $(n=211)$

\begin{tabular}{llllll}
\hline & All & No problems & Some problems & Large problems & Not driving \\
\hline No of cases & 211 & 21 & 78 & 9 & 92 \\
Mean age (years) & 70.8 & 74.5 & 67.7 & 61.3 & 73.2 \\
Age range (years) & $38-91$ & $65-91$ & $40-83$ & $46-86$ & $38-90$ \\
Males (\%) & 59 & 71 & 63 & 67 & 51 \\
First eye (\%) & 78 & 76 & 80 & 89 & 75 \\
Right eye (\%) & 51 & 48 & 58 & 44 & 50 \\
\hline
\end{tabular}

* 11 patients did not state their degree of visual problems while driving.

Table 2 Self estimated degree of visual functional problems while driving before surgery in relation to mean visual acuity (MVA) (SD) before and after surgery in operated, fellow, and better eye, respectively

\begin{tabular}{lllll}
\hline & No problems & Some problems & Large problems & Not driving \\
\hline $\begin{array}{l}\text { Before surgery: } \\
\quad \text { MVA eye to be }\end{array}$ & & & & \\
$\quad$ operated & $1.36(0.61)$ & $0.99(0.61)$ & $1.05(0.61)$ & $1.34(0.61)$ \\
$\quad$ MVA fellow eye & $0.15(0.19)^{\star}$ & $0.23(0.26)^{\star \star}$ & $0.21(0.22)$ & $0.44(0.47)$ \\
After surgery: & & & & \\
$\quad$ MVA operated eye & $0.06(0.06)^{\star \star \star}$ & $0.14(0.17)^{\star \star \star}$ & $0.23(0.33)^{\star \star \star}$ & $0.20(0.27)^{\star \star \star}$ \\
$\quad$ MVA better eye & $0.05(0.05)$ & $0.08(0.19)$ & $0.17(0.31)$ & $0.16(0.25)$ \\
\hline
\end{tabular}

$\star \star \star$ Significant improvement of MVA after surgery in the operated eye at a level of $p<0.0001$. $\star \star$ Significantly less improvement in MVA of the fellow eye before surgery for non-drivers compared with the various groups of drivers at a level of $\mathrm{p}<0.001$ and ${ }^{\star} \mathrm{p}<0.01$.

Table 3 Ratio of drivers (\%) after surgery in various age groups of cases with driving licences and differences between the sexes

\begin{tabular}{lccccl}
\hline Age (years) & \multicolumn{2}{c}{ All } & Males & Females & p Values \\
\hline-49 & $14 / 15(93)$ & $3 / 3(100)$ & $11 / 12(92)$ & $>0.1$ \\
$50-59$ & $14 / 16$ & $(88)$ & $8 / 8(100)$ & $6 / 8(75)$ & $>0.1$ \\
$60-69$ & $38 / 49(78)$ & $26 / 30(87)$ & $12 / 19(63)$ & $<0.05$ \\
$70-79$ & $58 / 86(67)$ & $41 / 50(82)$ & $17 / 36(47)$ & $<0.01$ \\
$80-$ & $13 / 45(29)$ & $11 / 34(32)$ & $2 / 11(18)$ & $>0.1$ \\
All ages & $137 / 211(65)$ & $89 / 125(71)$ & $48 / 86(56)$ & $<0.05$ \\
\hline
\end{tabular}

Table 4 Distance estimation problems in cases with driving licences

\begin{tabular}{lcccc}
\hline & \multicolumn{3}{c}{ Distance estimation problems } & \multirow{2}{*}{ No of patients } \\
\cline { 3 - 4 } & & Before surgery & After surgery & \multirow{2}{*}{ p Values } \\
\hline No problems & 21 & $0 \%(0 / 21)$ & $0 \%(0 / 21)$ & \\
Some problems & 78 & $44 \%(34 / 78)$ & $4 \%(3 / 78)$ & $<0.0001$ \\
Large problems & 9 & $100 \%(9 / 9)$ & $33 \%(3 / 9)$ & $<0.001$ \\
Not stated degree of problem & 11 & $9 \%(1 / 11)$ & $9 \%(1 / 11)$ & \\
Non-drivers before surgery & 92 & $58 \%(53 / 92)$ & $24 \%(22 / 92)$ & $<0.0001$ \\
All & 211 & $46 \%(97 / 211)$ & $14 \%(29 / 211)$ & $<0.0001$ \\
\hline
\end{tabular}

( $41 \%)$ were women. The median ages for men and women were 74 and 71 years, respectively. The distributions of driving licences for men and women in the different age groups are shown in Figures $1 \mathrm{~A}$ and B. In the subgroup of women older than 65 years of age $23 \%$ had driving licences. The corresponding figure for men was significantly higher $(78 \%$, $\mathrm{p}<0.00001)$. Below 55 years of age, women had driving licences as often as men.

Two hundred and forty eight surgical cases (54\%) had no driving licences. This group consisted of $87 \%$ women and only $13 \%$ men. The median ages for men and women in this group were 79.5 and 78.5 years, respectively.

PATIENTS WITH DRIVING LICENCES, THEIR VISUAL FUNCTIONAL PROBLEMS WITH DRIVING, AND THEIR VISUAL ACUITY

One hundred and nineteen (56\%) of the 211 cases with cataract and driving licences were drivers before surgery (Table 1). Of these 119 cases, $98(82 \%)$ said that they had visual func- tional problems with driving before surgery; 78 $(66 \%)$ stated they had some problems, nine cases $(8 \%)$ had large problems, and 11 cases (9\%) had not indicated the degree of their problems. Twenty one drivers (18\%) had no self estimated visual problems driving.

The most common visual problem specified was difficulty when driving in darkness and twilight. Seventy one per cent of the patients who specified their visual problems had such difficulties. Thirty seven per cent of the drivers experienced problems with distance estimation while driving; $11 \%$ were disturbed by glare while meeting other cars and $7 \%$ felt fatigue of their eyes while driving. The type of complaint between the group with 'some problems' and that with 'large problems' did not differ.

Before surgery no statistical correlation was found between the degree of self estimated visual functional problems and the mean visual acuity (MVA) in the eye to be operated on (Table 2). However, before surgery the MVA of the fellow eye for the patients who did not drive was significantly lower $(\log M A R=0.44)$ than in drivers with no and some visual functional problems $(\log M A R=0.15$ and $0.23, p<0.01$ and $\mathrm{p}<0.001$, respectively). Patients who drove before surgery with no visual problems had a somewhat better MVA $(\log M A R=0.15)$ in the fellow eye than the patients with problems (MVA=logMAR $0.21-0.23$ ). However, these differences were not statistically significant. A total of 164 surgical events $(78 \%)$ had been performed on the patients' first eye (Table 1); $51 \%$ performed on the right eye. There was no correlation between type and degree of visual functional problems and whether the right or the left eye or the first or the second eye was to be operated on.

The ratio between the patients who were drivers in relation to all with driving licences was $56 \%(119 / 211)$ before surgery. This ratio had increased to $65 \%(137 / 211)$ after surgery, but was not significant (Table 3 ). Five patients ceased driving after surgery. A significantly smaller ratio of women with licences were drivers after surgery compared with the men in the older age groups, $60-69$ years $(63 \%$ and $87 \%$ respectively, $\mathrm{p}<0.05)$ and $70-79$ years $(47 \%$ and $82 \%$ respectively, $p<0.01)$. Of those who were 70 years and older, 131 had driving licences; $61(47 \%)$ of these were drivers before surgery and $71(54 \%)$ after surgery (Table 3). However, this increase was not statistically significant.

After surgery there were remaining subjective visual functional problems in the operated eye in only $5 \%$ of the drivers (7/137), most often $(n=6)$ because of glare disability. A significant improvement in the MVA was observed after surgery in all groups with driving licences (Table 2). The visual acuity did not improve in four cases $(1.9 \%)$.

In Table 4 the cases with distance estimation problems while driving before and after surgery are presented. Before surgery $46 \%$ (97/211) of all cases with driving licences had problems in estimating distance. After surgery only $14 \%(29 / 211)$ had such problems $(\mathrm{p}<0.0001)$. This subgroup of 29 cases with 
remaining problems did not differ in age and sex from those without distance estimation problems. However, the MVA in their operated eyes $(\log M A R=0.28, p<0.01)$ and their fellow eyes $(\log M A R=0.54, p<0.001)$ were significantly lower. Furthermore, there were significantly $(\mathrm{p}<0.01)$ more cases with a VA of $\log$ MAR $>0.3(<20 / 40)$ in one eye among those with remaining problems $(62 \%, 18 / 29)$ compared with those without problems $(33 \%$, 60/182)

Among those who drove before surgery $37 \%$ (44/119) had distance estimation problems. After surgery this figure had significantly decreased to $5.9 \%(7 / 119 ; \mathrm{p}<0.0001)$.

Patients with no self estimated visual functional problems driving before surgery

Twenty one $(18 \%)$ of the drivers were not subjectively disturbed by their cataractous eyes before surgery (Table 1). This subgroup had not experienced any problems with distance evaluation either before or after surgery (Table 4). Three of them (14\%) did not fulfil the requirements for driving.

After surgery seven of these 21 cases (33\%) were driving more often. No one had ceased driving. All patients except one experienced unchanged or better subjective visual function after surgery. One patient (5\%) had problems with glare, diplopia, and fatigue of his eyes while driving after surgery as a result of large postoperative anisometropia (3.5 D).

Patients with some visual functional problems with driving before surgery

This subgroup consisted of 78 cases and they were significantly younger (mean age 67.7 years, $\mathrm{p}<0.05$ ) compared with the whole group of patients with driving licences (Table 1). Twenty three per cent (18/78) did not fulfil the visual acuity requirements before surgery.

Forty seven per cent $(37 / 78)$ of the patients were driving more often after surgery. Four patients $(5 \%)$ did not drive after surgery. Three of these patients did not drive because of a visual acuity that was too low $(\mathrm{VA}<20 / 40)$ and one because of old age.

Three patients (4\%) experienced visual functional problems after surgery, two cases because of glare sensitivity problems as a result of 'after-cataract'. The third patient had problems with distance estimation and felt fatigue of his eyes while driving; this might be the result of a four line difference in visual acuity between the eyes as one eye had age-related maculopathy.

The number of patients with distance estimation problems decreased significantly from $44 \%(34 / 78)$ before surgery to $4 \%(3 / 78)$ after surgery $(\mathrm{p}<0.00001$, Table 4$)$.

Patients with large visual functional problems with driving before surgery

This subgroup consisted of nine cases (Table 1). These patients were also significantly younger (mean age 61.3 years, $\mathrm{p}<0.01$ ) compared with the other patients. Two patients had a visual acuity below the requirements for driving before surgery.
Table 5 Drivers not fulfilling the visual requirements for driving in relation to age, sex, surgery on first eye and right eye, respectively, and mean visual acuity (MVA) before and after surgery in operated, fellow, and better eye, respectively

\begin{tabular}{lll}
\hline & Before surgery & After surgery \\
\hline No of cases: & $27^{\star}$ & 5 \\
Mean age (years) & 71.9 & 72.8 \\
Age range (years) & $52-86$ & $63-81$ \\
Males & $19 / 27(70 \%)$ & $4(80 \%)$ \\
First eye & $24 / 27(89 \%)$ & $4(80 \%)$ \\
Right eye (\%) & $10 / 27(37 \%)$ & $2(40 \%)$ \\
Before surgery (SD): & $0.94(0.45)$ & $1.12(0.54)$ \\
MVA eye to be operated & $0.63(0.28)$ & $0.87(0.32)$ \\
MVA fellow eye & & \\
After surgery (SD): & $0.25(0.25)^{\star \star \star}$ & $0.57(0.25)$ \\
MVA operated eye & $0.18(0.20)$ & $0.45(0.07)$ \\
MVA better eye &
\end{tabular}

$\star$ Four of these cases did not state their degree of visual problems while driving. ${ }^{\star} \star \star$ Significant improvement in MVA after surgery in the operated eye at a level of $p<0.0001$.

After surgery four patients drove more frequently and one patient did not drive because his visual acuity did not fulfil the requirements for driving. One patient experienced fatigue of his eyes and glare sensitivity problems especially when driving in twilight and darkness. This was probably due to surgical damage to the iris and a slightly decentred IOL.

All patients had problems with distance estimation before surgery (Table 4). After surgery only three patients had such problems, a significant decrease $(\mathrm{p}<0.01)$.

RELATION BETWEEN DRIVING AND FULFILMENT OF VISUAL REQUIREMENTS FOR DRIVING

Twenty seven surgical cases $(23 \%)$ out of a total of 119 who drove before surgery were found to have a visual acuity below what is required for driving in Sweden (Table 5); they comprised 19 men and eight women. The MVA of their operated eyes improved significantly from $\log M A R=0.94$ before surgery to $\log M A R=0.25$ after surgery $(p<0.0001)$. After surgery only five of 136 drivers $(3.7 \%)$ did not fulfil the requirements, which is a statistically significant decline $(p<0.0001)$. The patient group of 119 cases did not significantly differ in age and sex distribution, compared with the other patients with driving licences.

PATIENTS WITH DRIVING LICENCES WHO DID NOT DRIVE BEFORE BUT STARTED TO DRIVE AFTER SURGERY

This subgroup consisted of 23 patients (Table 6). Before surgery $39 \%$ (9/23) did not fulfil the visual requirements for driving. All these patients started to drive after surgery and had a visual acuity that fulfilled the requirements of driving. Two patients $(9 \%)$ complained of glare sensitivity after surgery.

PATIENTS WITH DRIVING LICENCES WHO DID NOT DRIVE BEFORE OR AFTER SURGERY

Sixty nine surgical cases did not drive either before or after surgery (Table 6). Before surgery 34 cases of these $(49 \%)$ did not fulfil the visual requirements for driving in Sweden.

Table 7 shows the reasons these patients did not start to drive after surgery. Twelve patients 
Table 6 Non-drivers before surgery and subjective reasons for not driving after surgery in relation to age, sex, surgery on first eye and right eye, respectively, and mean visual acuity (MVA) before and after surgery in operated, fellow, and better eye, respectively $(n=92)$

\begin{tabular}{|c|c|c|c|c|}
\hline & \multirow[b]{2}{*}{$\begin{array}{l}\text { Started to drive after } \\
\text { surgery }\end{array}$} & \multicolumn{3}{|c|}{ Not driving after surgery } \\
\hline & & All & $\begin{array}{l}\text { Subjectively too old } \\
\text { to drive }\end{array}$ & $\begin{array}{l}\text { Subjectively visual } \\
\text { function too bad }\end{array}$ \\
\hline No of cases: & 23 & 69 & 25 & 4 \\
\hline Mean age (years) & 64.0 & 76.0 & 77.2 & 55.8 \\
\hline Age range & $38-87$ & $44-90$ & $68-86$ & $47-66$ \\
\hline Males & $11 / 23(48 \%)$ & $36 / 69(52 \%)$ & $10 / 25(40 \%)$ & 0 \\
\hline First eye & $17 / 23(74 \%)$ & $51 / 69(74 \%)$ & $20 / 25(80 \%)$ & $4 / 4(100 \%)$ \\
\hline Right eye & $14 / 23(61 \%)$ & $34 / 69(49 \%)$ & $12 / 25(47 \%)$ & $1 / 4(25 \%)$ \\
\hline \multicolumn{5}{|l|}{ Before surgery: } \\
\hline MVA eye to be operated & $1.31(0.58)$ & $1.35(0.62)$ & $1.38(0.68)$ & $1.10(0.66)$ \\
\hline MVA fellow eye & $0.35(0.47)$ & $0.48(0.48)$ & $0.28(0.27)$ & $0.50(0.24)$ \\
\hline \multicolumn{5}{|l|}{ After surgery: } \\
\hline MVA operated eye & $0.09(0.12)$ & $0.24(0.30)$ & $0.11(0.11)$ & $0.06(0.07)$ \\
\hline MVA better eye & $0.05(0.06)$ & $0.19(0.28)$ & $0.07(0.07)$ & $0.06(0.07)$ \\
\hline
\end{tabular}

$(17 \%)$ did not fulfil the visual requirements after surgery.

Twenty five patients (36\%) had an excellent visual acuity (MVA=logMAR 0.07 ) after surgery and no other health problems (Table 6). However, they did not start to drive as they felt too old. In this group there were more women $(60 \%)$ than men $(40 \%)$, but this difference was not statistically significant. Their MVA of the operated eye after surgery was slightly lower ( $\log M A R=0.11)$ compared with those in the group who started to drive after surgery (0.09). This difference was not statistically significant $(\mathrm{p}=0.4)$.

Four patients $(6 \%)$ did not start to drive after surgery although fulfilling the visual requirements (Table 6). They felt their visual function was too bad for driving. This small subgroup consisted of comparatively younger women (mean age 55.8 years) with binocular cataract. All had very good visual acuities in their operated first eyes (MVA= $\log M A R \quad 0.06)$ after surgery. They had not started to drive because their non-operated cataractous eyes caused glare and subjective reduction of their visual fields.

\section{Discussion}

In the present study the benefit of cataract surgery for patients with driving licences is evident. Twenty five per cent (23/92) of the non-drivers before surgery started to drive after surgery. Forty per cent (48/119) of all drivers reported that they drove more frequently after surgery, and only 4\% (5/119) ceased driving.

Applegate et al studied cataract patients aged 70 years old and older. ${ }^{10}$ They found that $21 \%$ of the patients reported 'ability to drive' before surgery and $34 \%$ of the patients being 'able drivers' 1 year after surgery. We found $47 \%$

Table 7 Non-drivers after surgery and causes of not driving after surgery ( $n=69)$

\begin{tabular}{lc}
\hline & No of cases \\
\hline Visual acuity below driving requirements & $12(17 \%)$ \\
Healthy, but subjectively too old to drive & $25(36 \%)$ \\
Subjectively visual function too bad & $4(6 \%)$ \\
Limitations as a result of other diseases such as stroke, parkinsonism, & $24(35 \%)$ \\
$\quad$ cardiovascular complications & $2(3 \%)$ \\
Deceased & $2(3 \%)$ \\
No information & \\
\hline
\end{tabular}

driving before and $54 \%$ after surgery in the corresponding age group. The higher frequencies observed in our study can probably be explained by our patients having an increased need and urge to drive. In northern Sweden there are large geographic distances and relatively few means of public transport. Car driving is an important way of communication and many individuals are highly dependent on driving for their daily activities.

An important and new observation in the present study is that the patients' problems of estimating distance while driving was found to decrease significantly from $46 \%$ before surgery to $14 \%$ after surgery. Furthermore, significantly more patients with a visual acuity of less than 20/40 in one eye after surgery had such problems compared with those with an acuity of 20/40 or better in their worse eyes. This is an expected result since perception of distance is known to be correlated with and/or improved by an intact binocular vision. ${ }^{14}$ Moreover, distance estimation is a decisive function for optimal and safe car driving. ${ }^{15}$ Thus, our findings indicate that surgery of the fellow eye should be recommended if significant cataract is present, in order to obtain good binocular vision and achieve optimal ability for distance estimation.

Another notable finding in our study is that about a quarter of the patients were found to drive with a binocular visual acuity not fulfilling the legal requirements before surgery. This observation has not been reported previously to the authors' knowledge. The present finding is probably due to the often insidious decline in visual acuity caused by advancing cataract. The patients were simply unaware of the severity of their visual handicap. Extrapolating our results to all cataract patients in Sweden, approximately 1900 patients may be driving with a visual acuity below what is legally required. This number is probably overestimated, as by 1992, the frequency of cataract surgery was lower (3.3/1000 population) in our region than the average figure in Sweden (4.5/1000 population). ${ }^{16}$ It is well known that with increasing frequency of cataract surgery, the visual acuity of both eyes will rise at the time of the operation. ${ }^{17}$ Moreover, the interval from examination to surgery 
at our clinic is comparable with other clinics in Sweden. ${ }^{16}$ Cataract patients in northern Sweden are probably waiting longer before they request surgery. We suggest that patients with functional visual problems while driving should be considered for cataract surgery to reduce the number of patients driving with insufficient visual acuity and consequently lower the risk of traffic accidents. ${ }^{3} 18-20$

In the present study five patients $(4 \%)$ were found to be driving with insufficient visual acuity after surgery. In Sweden, unlike in some states of the USA, restricted licences for lower visual acuities than $20 / 40$ are not allowed. ${ }^{21}$ Therefore, it is important that cataract patients with a visual acuity below the requirements for driving are informed that their vision is insufficient. We suggest that this responsibility should be shared by ophthalmologists and opticians.

It is interesting to note that as many as one third $(33 \%)$ of patients with driving licences were found not to drive either before or after surgery. About one third (36\%) of them were found to be healthy elderly patients ( 68 years plus) who felt too old to drive in spite of an excellent visual acuity and no subjective visual problems. Women dominated $(60 \%)$ this group. This finding is in agreement with women generally being more cautious and less inclined to take risks. ${ }^{2022}$

We found about half (54\%) of the cataract patients did not have driving licences. These patients were mostly $(87 \%)$ elderly women. Only about a quarter $(23 \%)$ of women older than 65 years of age had licences whereas a majority $(78 \%)$ of the men of the same age group did so. However, below 55 years of age as many women as men held driving licences. This is presumably due to the fact that women, in the older age groups, by tradition have never learnt to drive.

In our study, surgery was found to have been performed on women in about two thirds of the cases. This finding is in accordance with women generally living longer, and being more numerous than men in the older age groups. ${ }^{11}$ It has also been shown that elderly women have a higher incidence of lens opacities than men of the same age. ${ }^{2324}$ Moreover, the elderly represent the most rapidly growing part of the driving population in industrialised societies. By the year 2024 one out of four drivers in Western Europe is estimated to be older than 65 years. $^{25}$ Thus, our findings, together with others, indicate that there will be an escalating request for cataract surgery as the population of elderly grows and more women will be driving.

A highly significant post-surgical increase of visual acuity was found in all patient groups in the present study. These results are comparable with earlier reports. ${ }^{78}$ The complication rate of zonular/capsular rupture was in the upper range of previous investigations. ${ }^{8}$ This is probably the result of two surgeons being relatively less experienced and, also because, at that time the phacoemulsification technique was being introduced to our clinic.

In summary, the present study shows that the majority of the car driving population benefits from cataract surgery in terms of subjectively improved visual function with distance estimation while driving as well as objectively augmented visual acuity. Secondly, about one quarter of the drivers scheduled for cataract surgery were driving while not fulfilling the visual acuity requirements. Thirdly, visual functional problems while driving should be considered as an indication for surgery. Finally, our findings indicate that the fellow eye should be operated, if necessary, to obtain good binocular vision and achieve optimal ability for distance estimation.

The authors thank Kerstin Johansson at the Swedish National Register of Driving Licences for providing data concerning driving licences. We also thank the nursing staff at the Eye Clinic of Norrlands University Hospital, Umeå, for distributing the questionnaires. Lennarth Nyström MSci, Department of Epidemiology and Public Health, Umeå University, is thanked for giving statistical advice.

1 Kline DW, Kline TJ, Fozard JL, Kosnik W, Schieber F, Sekuler R. Vision, aging and driving: the problems of older drivers. F Gerontol 1992;47:27-34

2 Ball K, Owsley C, Sloane ME, Roenker DL, Bruni JR. Visual attention problems as a predictor of vehicle crashes in older drivers. Invest Ophthalmol Vis Sci 1993;34:311023 .

3 Owsley C, Ball K, Sloane ME, Roenker DL, Bruni JR. Visual/cognitive correlates of vehicle accidents in older drivers. Psychol Aging 1991;6:403-15.

4 Johnson C, Keltner J. Incidence of visual field loss in 20,000 eyes and its relation to driving performance. Arch Ophthalmol 1986;101:371-5.

5 Olson P, Sivak PL. Perception-response time to unexpected hazards. Hum Factors 1986;28:91-6.

6 Retchin SM, Cox J, Fox M, Irwin L. Performance-based measurement among elderly drivers and non-drivers. $f \mathrm{Am}$ Geriatr Soc 1988;36:813-9.

7 Holland GN, Earl DT, Wheeler NC, Straatsma BR, Pettit TH, Hepler RS, et al. Results of inpatient and outpatient cataract surgery. Ophthalmology 1992;99:845-52.

8 Powe NR, Schein OD, Gieser SC, Tielsch JM, Luthra R, Javitt J, et al. Synthesis of the literature on visual acuity and complications following cataract extraction with IOL implantation. Arch Ophthalmol 1994;12:239-52.

9 Lasa MS, Datiles MB, Podgor MJ, Magno BV. Contrast and glare sensitivity. Ophthalmology 1992;99:1045-9.

10 Appelgate WB, Miller ST, Elam JT, Freeman JM, Wood TO, Gettlefinger TC. Impact of cataract surgery with lens implantation on vision and physical function in elderly patients. FAMA 1987;257:1064-6.

11 Official Statistics of Sweden. Folkmängden 31 December 1992. Liber, Stockholm: National Central Bureau of Statistics, 1993.

12 Mangione CM, Phillips RS, Seddon JM, Lawrence MG, Cook EF, Dailey R, et al. Development of the 'activities of daily vision scale'. A measure of visual functional status. Med Care 1992;30:1111-26.

13 Steinberg EP, Tielsch JM, Schein OD, Javitt JC, Sharkey P, Cassard SD, et al. The VF-14. An index of functional impairment in patients with cataract. Arch Ophthalmol 1994;112:630-8.

14 Cavallo V, Laurent M. Visual information and skill level in time-to-collision estimation. Perception 1988;17:623-32.

15 Keeney AH. Ophthalmic pathology in driver limitation. Trans Am Acad Ophthalmol Otolaryngol 1968;72:737-40.

16 Stenevi U, Lundström M, Thorburn W. A National Cataract Register. I. Description and epidemiology. Acta Ophthalmol (Scand) 1995;73:41-4.

17 Ninn-Pedersen K, Stenevi U, Ehinger B. Cataract patients in a defined Swedish population 1986-1990. II Preoperative observations. Acta Ophthalmol (Copenh) 1994;72:10-5.

18 Hills BL. Vision, visibility, and perception in driving. Perception 1980;9:183-216.

19 Shinar D, Schieber F. Visual requirements for safety and mobility of older drivers. Hum Factors 1991;33:507-19.

20 Kosnik WD, Sekuler R, Kline DW. Self-reported visual problems of older drivers. Hum Factors 1990;32:597-608.

1 Keltner JL, Johnson CA. Visual function, driving safety and the elderly. Ophthalmology 1987;94:1180-8.

22 Schiff W, Oldak R, Shah V. Aging persons' estimates of vehicular motion. Psychol Aging 1992;7:518-25.

23 Bishara SA, Goya V, Rand WJ. Cataract and ocular parameters: sexual comparison. Ann Ophthalmol 1988;20: 73-4.

24 Ninn-Pedersen K, Stenevi U, Ehinger B. Cataract patients in a defined Swedish population 1986-1990. I Resources in a defined Swedish population 1986-1990. I Resources

25 Transportation Research Board. Transportation in an aging society. Washington DC: National Research Council, 1988, vol 1 
Appendix

On the cover pages the aims of the questionnaires are presented and instructions on how to complete them are given to the patients.

\section{Questionnaire I}

BEFORE CATARACT SURGERY

1 Do you currently drive a car?

( ) No

() Yes

What degree of visual problems, if any, do you have driving?

Do you have:

( ) No problems

() Some problems

() Large problems

Please specify:

2 Do you experience difficulties estimating distance far away (that is, while driving (drivers) or in other 'traffic situations' (nondrivers))?

() Yes

() No

\section{Questionnaire II}

AFTER CATARACT SURGERY

1 Do you currently drive a car?

( ) No

( ) Yes

If yes, do you drive more often now than before surgery?

() Yes

( ) No

Do you experience that your visual function while driving has changed after your cataract operation?

( ) For the better

() No change

( ) For the worse

2 Do you experience difficulties to estimate distance far away (that is, while driving (drivers) or in other 'traffic situations' (nondrivers))?

( ) Yes

( ) No

3 Do you think that the operated eye disturbs your visual function while driving?

Please specify: 